\title{
Epidemiological Modeling of Bovine Brucellosis in India
}

\author{
Gloria J. Kang \\ Department of Population Health Sciences \\ Virginia Tech \\ Blacksburg, USA \\ gkang@,vt.edu \\ L. Gunaseelan \\ Department of Veterinary Public Health and Epidemiology \\ Madras Veterinary College \\ Tamil Nadu Veterinary and Animal Sciences University \\ Chennai, India \\ hodvphmvc@tanuvas.org.in
}

\author{
Kaja M. Abbas \\ Department of Population Health Sciences \\ Virginia Tech \\ Blacksburg, USA \\ kaja.abbas@vt.edu
}

\begin{abstract}
The study objective is to develop an epidemiological model of brucellosis transmission dynamics among cattle in India and to estimate the impact of different prevention and control strategies. The prevention and control strategies are test-and-slaughter, transmission rate reduction, and mass vaccination. We developed a mathematical model based on the susceptible-infectious-recovered epidemic model to simulate brucellosis transmission dynamics, calibrated to the endemically stable levels of bovine brucellosis prevalence of cattle in India. We analyzed the epidemiological benefit of different rates of reduced transmission and vaccination. Test-andslaughter is an effective strategy for elimination and eradication of brucellosis, but socio-cultural constraints forbid culling of cattle in India. Reducing transmission rates lowered the endemically stable levels of brucellosis prevalence correspondingly. One-time vaccination lowered prevalence initially but increased with influx of new susceptible births. While this epidemiological model is a basic representation of brucellosis transmission dynamics in India and constrained by limitations in surveillance data, this study illustrates the comparative epidemiological impact of different bovine brucellosis prevention and control strategies.
\end{abstract}

Keywords-brucellosis; epidemiology; India; mathematical model; prevention and control strategies; vaccination

\section{INTRODUCTION}

Brucellosis is a complex zoonotic disease with significant epidemiological, economic, and global health impact, particularly for human and animal populations within developing countries that rely on cooperative farming and agricultural practices [1], [2]. Evidence of the changing ecology and reemergence of brucellosis over recent years has demonstrated the pathogen's ability to seamlessly and rapidly adapt to the modern world, necessitating innovative approaches to epidemiological study and intervention design [3].

\section{A. Brucellosis microbiology}

Brucellosis is caused by facultative, intracellular bacteria of genus Brucella that survive and reproduce within host phagocytic cells. The pathogenicity of Brucella spp. involves an efficient adaptation that prevents recognition by the immune system and manipulates fundamental properties of host cell physiology [4]. However, current knowledge of the bacterium's pathogenic processes and biology is limited. The lack of classic virulence factors, such as capsules, plasmids, pili, or exotoxins, suggest the presence of unique mechanisms by which cell invasion and immune system evasion occur [5]. Brucellosis represents a significant threat to the future of public health given evidence of highly persistent novel strains with the ability to reemerge in new species and environmental foci.

\section{B. Brucellosis transmission dynamics}

In humans, transmission generally occurs as a result of ingestion of infected milk products or direct contact with infectious material through broken skin. Human brucellosis most often presents as an acute or sub-acute febrile disease typically marked with a cycling, undulant fever. Because symptoms commonly mimic other endemic diseases such as malaria, typhoid, and influenza, misdiagnosis occurs [6]. The disease is rarely fatal, but various clinical manifestations tending towards chronicity and persistence can lead to severely debilitating and disabling complications within any organ system [5].

In animals, transmission of bovine brucellosis, caused by Brucella abortus in cattle, occurs through ingestion of infected tissues or body fluids, contact with mucous membranes, direct inoculation, and fomites. The characteristic symptoms of infection in cattle are spontaneous abortion and premature birth with retained placenta. However, these symptoms are not specific to brucellosis, thereby contributing to misdiagnosis and misreporting of disease. In contrast, infections can also be asymptomatic and exist in healthy livestock [7]. In addition to the zoonotic threat of spillover into human populations, infected herds can cause significant losses to developing economies dependent on agrarian practices such as livestock herding and milk production.

\section{Brucellosis epidemiology in India}

In India, about $80 \%$ of people live within close contact to domestic livestock animals or wildlife, a critical risk factor for zoonotic disease transmission such as brucellosis; yet, the true incidence of human brucellosis is unknown [8]-[10]. Seroprevalence studies suggest infection may range between $0.9 \%-18.1 \%$, with higher risk in veterinarians and farm attenders. However, misdiagnosis occurs due to differential diagnoses of malaria, typhoid, paratyphoid, and influenza. 
Brucellosis prevalence among livestock animals varies widely across India's diverse agro-ecological landscape, differing from region to region and farm to farm [9]. Senthil et al conducted a seroprevalence study of bovine brucellosis in Chennai, India utilizing various diagnostic tests with positive results ranging from $3.3 \%-11.4 \%$ [11]. Isloor et al found overall prevalence was $1.9 \%$ in cattle and $1.8 \%$ in buffalo [12]. Long-term serological studies indicate baseline seroprevalence of $5 \%$ in cattle and $3 \%$ in buffalo [6].

Progress reports of monitoring programs from 2012-2013 by the Indian Council of Agricultural Research estimate that the current national seroprevalence of brucellosis in cattle is roughly $13.5 \%$ and at a stable, endemic equilibrium [13]. While population seropositivity is generally a poor measure of disease, due to high levels of underreporting and insufficient data, we considered seroprevalence to be a proxy measure for brucellosis prevalence in this study.

\section{Brucellosis control and prevention}

There is no vaccine for humans, and high rates of initial treatment failure and relapse rates make clinical management particularly difficult [14]. Hence, prevention of human brucellosis depends on management of the animal reservoir. Disease management for brucellosis may best take the form of test-and-slaughter, abortion notification, and vaccination [15].

However, economic feasibility and efficacy must be taken into consideration depending on social, political, and ecological factors. Due to India's governmental ban on cattle slaughter, proposals for a brucellosis control and prevention program in India highlight a mass vaccination campaign within areas of high disease prevalence. Vaccination against bovine brucellosis in cattle is currently limited to large, organizedprivate, state-owned and military dairy farms [16].

\section{TABLE I. PUBLIC HEALTH POLICYAND PRACTICE QUESTIONS}

- What is the epidemiological impact of brucellosis: prevalence, incidence, mortality, morbidity?

- What is the geographic/spatial distribution of brucellosis?

- What is the demographic distribution of brucellosis?

- How reliable is surveillance of brucellosis?

- How to conduct hypothesis testing of different scenarios for risk/potential for spread and transmission dynamics?

- What interventions are warranted for prevention and control of brucellosis?

- When to introduce the interventions?

- What is the quantum and period of interventions?

Questions illustrate the public health policy and practice challenges faced by decision-makers in implementation and scale-up of the brucellosis control and prevention program in India.

\section{E. Public health significance}

Enhanced spread of brucellosis through increased distribution of potentially infected animal products poses significant social, economic, and public health threats unimpeded by political borders, demanding effective livestock disease management practices through the unified efforts of scientists, health professionals, and policy-makers. Effective development and implementation of a brucellosis prevention and control program in India can be facilitated by identifying answers to the public health policy and practice questions facing decision-makers, as illustrated in Table I. The public health significance of this study is to develop an epidemiological model of brucellosis transmission dynamics in India to address answers to these questions.

\section{METHODS}

Mathematical modeling of infectious disease dynamics provides an efficient environment to design and test intervention policies in a virtual computational framework [17]-[21].

\section{A. Epidemic model}

We developed a system dynamics model of bovine brucellosis transmission dynamics, as illustrated in Fig. 1. The cattle population is divided into three subpopulations: susceptible, infected/infectious, and immune. New births flow into the susceptible population, and natural deaths flow out of all compartments. As suceptible cattle are vaccinated effectively, these cattle move into the immune compartment. We assume no recovery from infection, no waning vaccination immunity, and no disease-induced mortality.

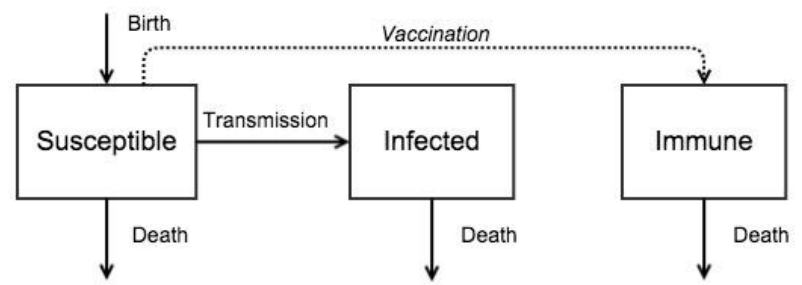

Fig. 1. System dynamics model of brucellosis transmission dynamics with births and deaths. The mass action mixing of susceptible and infected cattle lead to new infections. At the time of vaccination, susceptible cattle who are vaccinated effectively move to the immune compartment.

The ordinary differential equations for the SusceptibleInfected/Infectious-Recovered (SIR) epidemic model of brucellosis transmission dynamics are listed below.

$$
\begin{aligned}
& S_{0}=S_{0}-p v S_{0} \\
& \frac{d S}{d t}=\mu-\beta S I-\mu S \\
& \frac{d I}{d t}=\beta S I-\mu I \\
& \frac{d R}{d t}=p v S_{0}-\mu R
\end{aligned}
$$

The birth and death rates are uniformly assigned to $\mu$ due to the relatively stable population size of cattle in India, and $\beta$ represents the transmission rate. We adapted the SIR epidemic model to incorporate a one-time vaccination program by correspondingly changing the initial proportion of suceptibles $\left(S_{0}\right)$ and immune cattle. Susceptible cattle who gain immunity to brucellosis move into the immune $(R)$ compartment which depends on the vaccine efficacy $(v)$ and the proportion vaccinated $(p)$. 


\section{B. Data}

Data was obtained from Madras Veterinary College (MVC) in Chennai, India where the university research farms conduct ongoing studies regarding production and reproduction of various cattle breeds under the specific agro-climatic conditions of Tamil Nadu. Theses and dissertations from MVC and regional disease monitoring reports from the Project Directorate on Animal Disease Monitoring and Surveillance provided current seroprevalence of bovine brucellosis in cattle. Publically available livestock census data from the Ministry of Agriculture and Department of Animal Husbandry Dairying \& Fisheries provided birth and death rate parameterization for our model. The initial conditions of the epidemic model were set to an endemically stable level of $13.5 \%$ brucellosis prevalence for cattle in India.

\section{Simulation model}

The epidemic model is simulated in $R$ statistical software and programming environment [22]. Model parameters and corresponding values are shown in Table II.

TABLE II. MODEL PARAMETERS AND VALUES

\begin{tabular}{|l|c|}
\hline \multicolumn{1}{|c|}{ Parameter } & Value \\
\hline Susceptible proportion $(S)$ - endemic stability & 0.865 \\
\hline Infected proportion $(I)$ - endemic stability & 0.135 \\
\hline Transmission rate $(\beta)$ & $0.1156 /$ year \\
\hline Birth/Death rates $(\mu)$ & $0.1 /$ year \\
\hline Vaccine efficacy $(v)$ & $70 \%$ \\
\hline Proportion vaccinated $(p)$ & $0-100 \%$ \\
\hline
\end{tabular}

Model parameters reflect brucellosis transmission at endemic stability, with $13.5 \%$ infected cattle and $86.5 \%$ susceptible cattle in the population. The transmission rate $(\beta)$ is estimated at endemic stability. Birth and death rates are assigned to be uniform, corresponding to the stable population size and average life expectancy of 10 years. Vaccine efficacy reflects the reported protection of S19 vaccine - the B. abortus vaccine used in India.

\section{RESULTS}

We analyzed the brucellosis transmission dynamics model at endemic stability for control and prevention policies of testand-slaughter, reduction of transmission rates, and implementation of a one-time vaccination of cattle.

\section{A. Endemic stability}

Transmission of bovine brucellosis is estimated to occur at an endemic equilibrium in India, with disease prevalence estimated to be $13.5 \%$. Equilibrium dynamics were used to estimate the transmission rate of 0.1156 per year. Because the effective reproductive rate $\left(R_{0}\right)$ at the endemic stable state is 1 , effective control and prevention policies will lower $R_{0}$ below 1 , thereby shifting the transmission dynamics towards elimination of brucellosis.

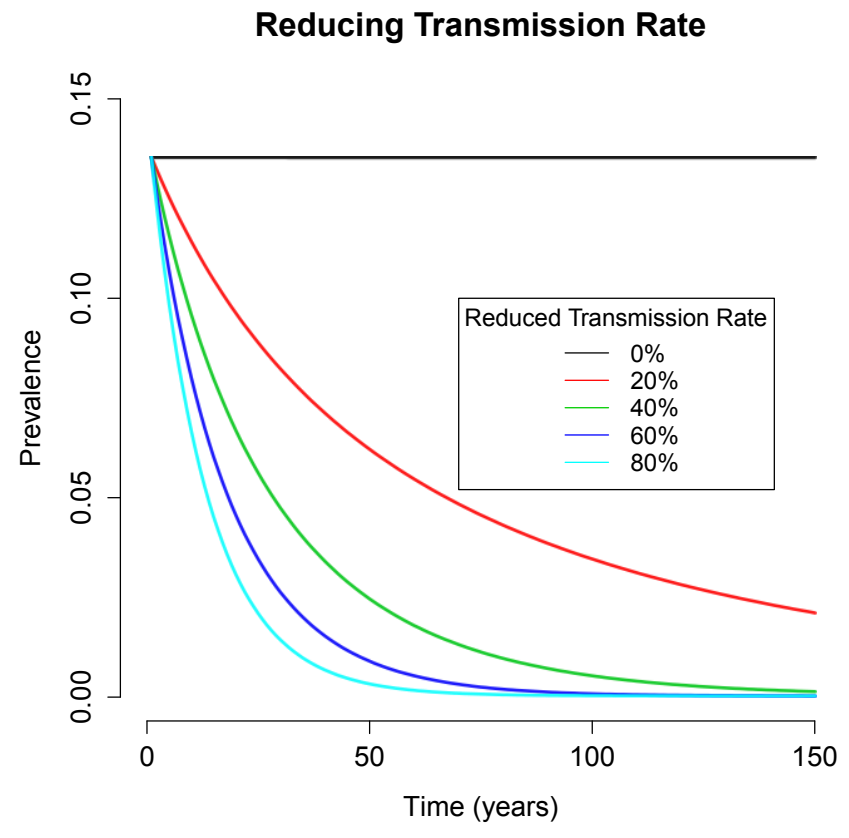

Fig. 2. Epidemiological impact of reducing transmission rate. Brucellosis prevalence decreases over time by reducing transmission rate by $20 \%, 40 \%$, $60 \%$, and $80 \%$ below the transmission rate at endemic stability (refers to $0 \%$ reduced transmission rate).

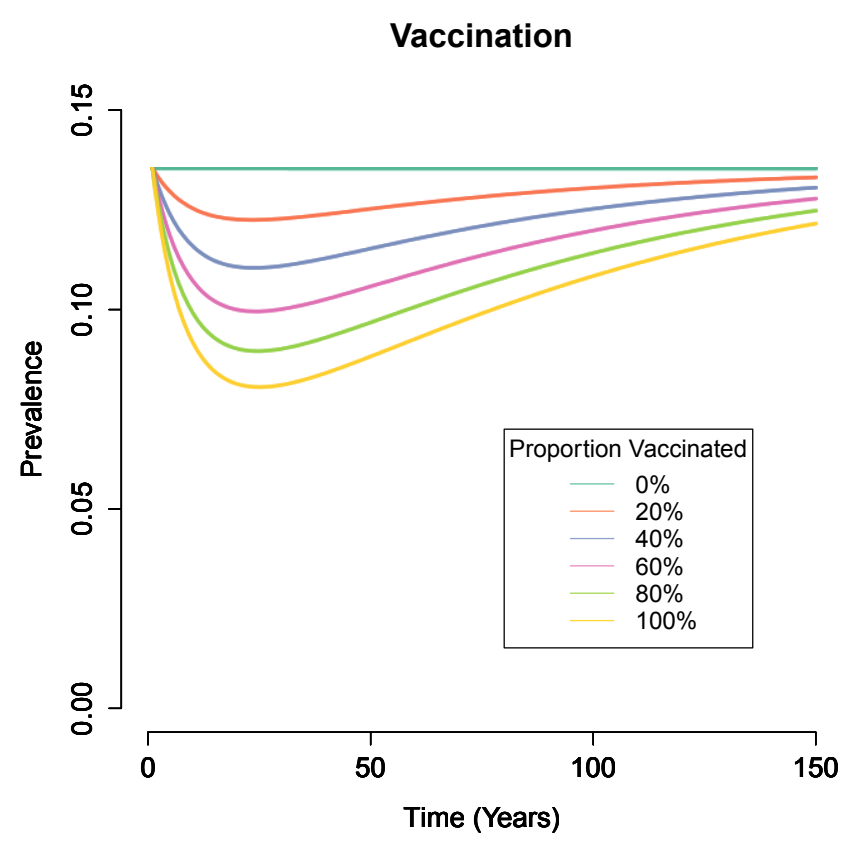

Fig. 3. Epidemiological impact of one-time vaccination. Brucellosis prevalence dynamics for one-time vaccination of $20 \%, 40 \%, 60 \%, 80 \%$, and $100 \%$ of the cattle population. 


\section{B. Test and slaughter}

Test and slaughter is a proven strategy for brucellosis elimination by culling and removing infected cattle from the population. The removal of infected cattle from the population eliminates onward transmission, thereby leading to near eradication. The success of this strategy depends on the sensitivity and specificity of the diagnostic tests and the proportion of population tested. Successful implementation of the brucellosis eradication program in United States involved large culls of cattle population, and has led to elimination and effective control of bovine brucellosis [23]. In India however, socio-cultural constraints forbid culling of cattle except for two states, thereby prohibiting actual implementation of the test and slaughter strategy.

\section{Reducing transmission rate}

We tested the prevention and control strategy of reducing transmission, by reducing the contact rate between susceptible and infected cattle. Fig. 2 illustrates the epidemiological impact of reducing transmission rate by $20 \%, 40 \%, 60 \%$, and $80 \%$; brucellosis prevalence is reduced and recedes towards elimination and eradication. Reducing transmission rate by $0 \%$ refers to the endemic stable state of brucellosis prevalence of $13.5 \%$.

\section{Vaccination}

Mass vaccination is an effective and efficient strategy to control and prevent brucellosis in animals, especially for cattle [24]. We tested a one-time vaccination at different rates of vaccination coverage with a vaccine efficacy of $70 \%$, the reported minimum efficacy of the S19 vaccine available in India [25]. Null vaccination coverage of $0 \%$ illustrates the current endemic stability scenario of $13.5 \%$ disease prevalence. The one-time vaccination program decreased brucellosis prevalence, with correspondingly higher benefits observed at higher rates of vaccination coverage of $20 \%, 40 \%, 60 \%, 80 \%$ and $100 \%$, as shown in Fig. 3.

After the one-time vaccination program, the influx of newly susceptible cattle through births into the population leads to a rebound of brucellosis toward the initial level of endemic prevalence. Further, brucellosis prevalence does not recede towards elimination and eradication even at vaccination coverage levels of $100 \%$.

\section{DISCUSSION}

We developed a basic epidemiological model of bovine brucellosis transmission dynamics for livestock cattle in India. We used this model to analyze the impact of different control and prevention strategies to reduce prevalence of brucellosis from the endemically stable state of $13.5 \%$ towards elimination and eradication. While test and slaughter is an effective disease management strategy, the sociocultural belief of cattle sanctity in India prohibit its implementation.

\section{A. Reducing transmission rate}

Reducing transmission rates by limiting host contact is an effective strategy for eliminating and eradicating bovine brucellosis, yet challenges remain in identifying feasible and effective practices to prevent ingestion of infected tissues and body fluids, as well as contact with mucous membranes and fomites in the environment. Strategies include physically limiting within-herd and between-herd contact patterns, either by reducing the frequency of interactions or the density of the cattle herd population.

\section{B. Vaccination}

A one-time vaccination program is an effective strategy to decrease the rate of new infections. By assuming a stable population size where birth and death rates are equal, we produced a conservative scenario in which a one-time vaccination of $100 \%$ of the population fails to sustain protective effects for a prolonged period due to the influx of the susceptible cattle through births. Thereby, vaccination programs must be implemented at regular intervals to prevent the rebound of brucellosis prevalence to initial endemic stability levels.

\section{Cattle population and demography}

Data from the Indian cattle census from 1997-2012 indicate a rapid increase in cattle population in the early years, followed by a plateau in growth rate in recent years. However, annual growth rates vary widely across states and among different cattle breeds, sex, and age, as does reported seroprevalence. The model inferences are a country-level estimation of bovine brucellosis epidemiological impact in India, and the results may not reflect the epidemiological dynamics within individual farms.

\section{Limitations}

The limitations of this study are the use of a basic epidemic model and simplified assumptions to derive the model parameters. The assumptions are necessitated due to the uncertainty in transmission dynamics of brucellosis and cattle demography. With respect to the cattle host, variations in transmission rate by age and gender is not included. With respect to the Brucella pathogen and immune dynamics, latent period and waning immunity is not included. With respect to environment, indirect transmission through an environmental reservoir is not considered. B. abortus diagnostic tests increase uncertainty and misdiagnosis, depending on sensitivity and specificity of the tests.

\section{E. Public health implications}

Despite the limitations and simplicities of this study, our model demonstrates the fundamental processes within bovine brucellosis transmission and the resulting effects of various intervention strategies in India. Mathematical models provide invaluable insight into the epidemiology of economicallyimportant diseases, particularly in developing countries where conventional methods of disease control are near-impossible to implement. With inevitable increases in human population, international travel, and economic demand, the potential global impact for a complex zoonosis like brucellosis goes beyond the realm of public health [26]. Hence, from a public health perspective, the management and prevention of brucellosis is a critical task that depends on effective communication with policy-makers. Our model provides 
partial solutions to the public health policy and practice questions that are listed in Table I, and have a meaningful discussion with decision makers on control and prevention of bovine brucellosis.

\section{F. Future work}

While our focus on country-wide brucellosis status provides a general understanding of brucellosis transmission dynamics across India, this model may not be representative of the average cattle farm.

Further studies modeling within-farm and between-farm transmission can assess individual contact patterns and determine localized disease dynamics. Spatially detailed models, such as network and agent-based models, can help define local public health needs at the community level [27].

We will refine the spatial focus to a local region or farm with better availability of data on brucellosis epidemiology. The variations in risk of Brucella acquisition and transmission by age and gender can be incorporated into the model, as well as indirect transmission through the environment. Uncertainty and sensitivity analysis will assist in calibration, verification and validation of brucellosis transmission dynamics.

\section{G. Conclusion}

Bovine brucellosis data in India is limited to studies at various time points across diverse geographic regions, thereby limiting our understanding of transmission dynamics and risk factors. However, by utilizing a systems-based approach of studying transmission dynamics, mathematical models allow for better understanding, analysis, and improvement of prevention and control strategies at the population level, especially for resource-limited areas.

The dynamic processes within zoonotic disease transmission, such as brucellosis, demands a "One Health" approach [26]. Transdisciplinary collaboration among the fields of human, animal, and environmental health sciences is critical in addressing the intersectoral nature that shape health, economic, and social policy worldwide. Improving our understanding of zoonotic disease ecology will be critical in the face of global change and population growth, and the prospects of mathematical modeling delivers an effective tool towards this imperative.

\section{ACKNOWLEDGEMENTS}

We thank the students, faculty and scientists of the Department of Veterinary Public Health and Epidemiology at Madras Veterinary College for their assistance and feedback on this study.

\section{REFERENCES}

[1] N. Haque, M. S. Bari, M. A. Hossain, N. Muhammad, S. Ahmed, A. Rahman, S. M. Hoque, and A. Islam, "An overview of Brucellosis," Mymensingh Med. J. MMJ, vol. 20, no. 4, pp. 742-747, Oct. 2011.

[2] E. Moreno, "Retrospective and prospective perspectives on zoonotic brucellosis," Front. Microbiol., vol. 5, p. 213, 2014.

[3] G. Pappas, "The changing Brucella ecology: novel reservoirs, new threats," Int. J. Antimicrob. Agents, vol. 36, pp. S8-S11, Nov. 2010.
[4] J. Godfroid, H. C. Scholz, T. Barbier, C. Nicolas, P. Wattiau, D. Fretin, A. M. Whatmore, A. Cloeckaert, J. M. Blasco, I. Moriyon, C. Saegerman, J. B. Muma, S. Al Dahouk, H. Neubauer, and J.-J. Letesson, "Brucellosis at the animal/ecosystem/human interface at the beginning of the 21st century," Prev. Vet. Med., vol. 102, no. 2, pp. 118-131, Nov. 2011

[5] M. P. Franco, M. Mulder, R. H. Gilman, and H. L. Smits, "Human brucellosis," Lancet Infect. Dis., vol. 7, no. 12, pp. 775-786, Dec. 2007.

[6] G. J. Renukaradhya, S. Isloor, and M. Rajasekhar, "Epidemiology, zoonotic aspects, vaccination and control/eradication of brucellosis in India," Vet. Microbiol., vol. 90, no. 1-4, pp. 183-195, Dec. 2002.

[7] M. N. Seleem, S. M. Boyle, and N. Sriranganathan, "Brucellosis: a reemerging zoonosis," Vet. Microbiol., vol. 140, no. 3-4, pp. 392-398, Jan. 2010.

[8] A. S. Agasthya, S. Isloor, and K. Prabhudas, "Brucellosis in high risk group individuals," Indian J. Med. Microbiol., vol. 25, no. 1, pp. 28-31, Jan. 2007.

[9] B. G. Mantur and S. K. Amarnath, "Brucellosis in India - a review," $J$. Biosci., vol. 33, no. 4, pp. 539-547, Nov. 2008.

[10] H. L. Smits and S. M. Kadri, "Brucellosis in India: a deceptive infectious disease," Indian J. Med. Res., vol. 122, no. 5, pp. 375-384, Nov. 2005.

[11] N. R. Senthil and S. A. Narayanan, "Seroprevalence Study of Bovine Brucellosis in Slaughter House," Int. J. Adv. Vet. Sci. Technol., vol. 2, no. 1, pp. pp. 61-63, Aug. 2013.

[12] S. Isloor, G. J. Renukaradhya, and M. Rajasekhar, "A serological survey of bovine brucellosis in India," Rev. Sci. Tech. Int. Off. Epizoot., vol. 17, no. 3, pp. 781-785, Dec. 1998.

[13] H. Rahman, "DBT Network Project on Brucellosis," Indian Council of Agricultural Research, Project Monitoring Unit, Project Directorate on Animal Disease Monitoring and Surveillance, Annual Report, Sep. 2013.

[14] P. M. Rabinowitz and L. A. Conti, Human-Animal Medicine: Clinical Approaches to Zoonoses, Toxicants and Other Shared Health Risks. Elsevier Health Sciences, 2009.

[15] S. Olsen and F. Tatum, "Bovine brucellosis," Vet. Clin. North Am. Food Anim. Pract., vol. 26, no. 1, pp. 15-27, table of contents, Mar. 2010.

[16] G. J. Renukaradhya, S. Isloor, and M. Rajasekhar, "Epidemiology, zoonotic aspects, vaccination and control/eradication of brucellosis in India," Vet. Microbiol., vol. 90, no. 1-4, pp. 183-195, Dec. 2002.

[17] E. Vynnycky and R. White, An Introduction to Infectious Disease Modelling, 1st ed. Oxford University Press, USA, 2010.

[18] H. McCallum, N. Barlow, and J. Hone, "How should pathogen transmission be modelled?," Trends Ecol. Evol., vol. 16, no. 6, pp. 295300, Jun. 2001.

[19] M. J. Keeling and P. Rohani, Modeling Infectious Diseases in Humans and Animals, 1st ed. Princeton University Press, 2007.

[20] S. S. Morse, J. A. K. Mazet, M. Woolhouse, C. R. Parrish, D. Carroll, W. B. Karesh, C. Zambrana-Torrelio, W. I. Lipkin, and P. Daszak, "Prediction and prevention of the next pandemic zoonosis," Lancet, vol. 380, no. 9857, pp. 1956-1965, Dec. 2012.

[21] H. V. Fineberg and M. E. Wilson, "Epidemic science in real time," Science, vol. 324, no. 5930, p. 987, May 2009.

[22] R, The R Project for Statistical Computing. http://www.r-project.org/, 2014.

[23] E. D. Ebel, M. S. Williams, and S. M. Tomlinson, "Estimating herd prevalence of bovine brucellosis in 46 USA states using slaughter surveillance," Prev. Vet. Med., vol. 85, no. 3-4, pp. 295-316, Jul. 2008.

[24] P. Nicoletti, "Brucellosis: past, present and future," Pril. Makedon. Akad. Na Nauk. Umet. Oddelenie Za Biološki Med. Nauki Contrib. Maced. Acad. Sci. Arts Sect. Biol. Med. Sci., vol. 31, no. 1, pp. 21-32, Jul. 2010.

[25] P. Nicoletti, "Vaccination against Brucella," Adv. Biotechnol. Processes, vol. 13, pp. 147-168, 1990.

[26] World Bank, "People, Pathogens and Our Planet." World Bank, 2010.

[27] D. A. Luke and K. A. Stamatakis, "Systems Science Methods in Public Health: Dynamics, Networks, and Agents," Annu. Rev. Public Health, Apr. 2011. 